\title{
Population Genomic Analyses Reveal Connectivity via Human-Mediated Transport across Populus Plantations in North America and an Undescribed Subpopulation of Sphaerulina musiva
}

\author{
J. F. Tabima, ${ }^{1}$ K. L. Søndreli, ${ }^{1}$ S. Keriö, ${ }^{1}$ N. Feau, ${ }^{2}$ M. L. Sakalidis, ${ }^{3}$ R. C. Hamelin, ${ }^{2}$ and J. M. LeBoldus ${ }^{1,4, \dagger}$ \\ ${ }^{1}$ Department of Botany and Plant Pathology, College of Agricultural Sciences, Oregon State University, Corvallis, U.S.A. \\ ${ }^{2}$ Department of Forest and Conservation Sciences, Faculty of Forestry, University of British Columbia, Canada \\ ${ }^{3}$ Department of Plant, Soil and Microbial Sciences and the Department of Forestry, College of Agriculture \& Natural Resources, \\ Michigan State University, East Lansing, U.S.A. \\ ${ }^{4}$ Department of Forest Engineering, Resources and Management, College of Forestry, Oregon State University
}

Accepted 1 October 2019.

\begin{abstract}
Domestication of plant species has affected the evolutionary dynamics of plant pathogens in agriculture and forestry. A model system for studying the consequences of plant domestication on the evolution of an emergent plant disease is the fungal pathogen Sphaerulina musiva. This ascomycete causes leaf spot and stem canker disease of Populus spp. and their hybrids. A population genomics approach was used to determine the degree of population structure and evidence for selection on the North American population of $S$. musiva. In total, 122 samples of the fungus were genotyped identifying 120,016 single-nucleotide polymorphisms after quality filtering. In North America, $S$. musiva has low to moderate degrees of differentiation among locations. Three main genetic clusters were detected: southeastern United States, midwestern United States and Canada, and a new British Columbia cluster (BC2). Population genomics suggest that $\mathrm{BC2}$ is a novel genetic cluster from central British Columbia, clearly differentiated from previously reported $S$. musiva from coastal British Columbia, and the product of a single migration event. Phenotypic measurements from greenhouse experiments indicate lower aggressiveness of BC2 on Populus trichocarpa. In summary, $S$. musiva has geographic structure across broad regions indicative of gene flow among clusters. The interconnectedness of the North American $S$. musiva populations across large geographic distances further supports the hypothesis of anthropogenic-facilitated transport of the pathogen.
\end{abstract}

${ }^{\dagger}$ Corresponding author: J. M. LeBoldus; jared.leboldus@science.oregonstate.edu

Funding: This study was supported by Department of Energy Office of Science, Office of Biological and Environmental Research Grant DESC0018196 to J. M. LeBoldus.

*The $\boldsymbol{e}$-Xtra logo stands for "electronic extra" and indicates that four supplementary figures and two supplementary tables are published online.

The author(s) declare no conflict of interest.

๑) 2020 The American Phytopathological Society
Keywords: genomics, microbial ecology, population biology, population genetics

Populus spp. and their interspecific hybrids are among the fastest-growing tree species in North America. As such, they represent a source of fiber and feedstock for lignocellulosic biomass. Populus spp. are ideal for this due to their ease of clonal propagation, broad geographic distribution, and wide range of environmental conditions to which they are adapted. In addition, Populus trees can be grown on economically marginal lands, eliminating competition with food production, a common concern with many biofuel feedstocks. As a result, plantations have been established across North America and around the world (Stanturf and Zhang 2003). As a direct result of this expansion, plant material has been freely exchanged among public and private breeding programs around the world. In many cases, the associated microbial communities have also likely been exchanged (Feau et al. 2010; Sakalidis et al. 2016).

The movement of Populus-associated microbes is perhaps best illustrated by Sphaerulina musiva Peck (Quaedvl., Verkley \& Crous; syn: Septoria musiva). Sphaerulina musiva is a heterothallic ascomycete that reproduces sexually and asexually. The winter-spring cycle is characterized by the production and dispersal of meiospores called ascospores formed in fruit bodies called perithecia, mostly on leaf litter (Bier 1939). The summer cycle is dominated by the spread of mitospores called conidia, produced by asexual reproduction in fruit bodies called pycnidia produced on symptomatic tissue (Waterman 1955). Both ascospores and conidia are responsible for disease in susceptible hosts. Ascospores serve as primary inoculum and conidia are the secondary inoculum. S. musiva was first reported as a leaf spot of Populus deltoides W. Bartram ex Marsh. in the eastern United States (Peck 1881) and, later, following introduction of Populus trees from the Tacamahaca botanical section, as a stem and branch canker of exotic and hybrid poplar across the Northern Great Plains (Bier 1939; Waterman 1946). Anthropogenic-facilitated movement is widely regarded as the most likely mode of S. musiva spread (Abraham et al. 2018; Herath et al. 2016; Sakalidis et al. 2016) and has resulted in the introduction of $S$. musiva into the Pacific Northwest of North America (Abraham et al. 2018; Callan et al. 2007, 2007; Feau 
et al. 2010; Sakalidis et al. 2016) and South America (Santos et al. 2010; Sarasola 1944). Once established, the impact of Septoria canker on susceptible hosts is predictably severe.

Septoria leaf spot and stem canker caused by $S$. musiva can lead to premature defoliation, branch and stem breakage, and increased mortality (Waterman 1955). The incidence of Septoria canker in Populus plantations can reach 100\% (LeBoldus et al. 2009) and, in many cases, result in plantation failure (Ostry and McNabb 1985, 1990). Efforts to manage this disease have included cultural (Ostry et al. 1989), chemical (Ostry and McNabb 1990), and biological control (Feau et al. 2010; Gyenis et al. 2003). These methods have proven to be largely ineffective or too expensive to be used over the rotation of a plantation (12 to 20 years). As a result, disease-resistant genotypes are widely regarded as the most effective way to manage this disease (LeBoldus et al. 2009; Ostry et al. 1989; Qin and LeBoldus 2014). The sequencing and annotation of the P. trichocarpa genome (Tuskan et al. 2006), has accelerated the search for resistance and led to the first identification of genes mediating the interaction with S. musiva (Muchero et al. 2018).

Many different Populus spp. and hybrids have been used to establish clusters of geographically isolated plantations across North America. These species include $P$. deltoides (native in eastern and north-central North America), P. trichocarpa (native in the Pacific Northwest), and P. balsamifera (transcontinental across northern the United States, Canada, and Alaska). This diversity has the potential to structure the pathogen population, leading to differences among geographic areas. Host genotype has been demonstrated to structure microbial populations in both agricultural and natural ecosystems (García-Palacios et al. 2013; Zeder 2008). For example, the migration-adaptation dynamics reported in Magnaporthe oryzae (Couch et al. 2005) have resulted in the expansion of the pathogen to many economically important species after a host shift to rice (Oryza sativa). This has resulted in local adaptation and population structure as a result of differences in geographic distribution and host (Couch et al. 2005). Plant domestication is fundamental in shaping the evolutionary dynamics of an emerging plant pathogen, because it provides a new environment for the pathogen to adapt and facilitates its expansion across distances beyond the range of the pathogen's dispersal (Giraud et al. 2010). However, no population-level genomic analysis has been performed to analyze the interplay between local adaptation, migration, and genetic drift for the areas where Populus tree cultivation is extensive.

Based on the influence of Populus domestication for human use, our hypothesis predicts that $S$. musiva populations are following a migration-adaptation scenario similar to $M$. oryzae (Couch et al. 2005), where $S$. musiva evolves under the selective pressures of each isolated Populus plantation following migration. This will result in local adaptation, natural selection, and highly structured populations of $S$. musiva within geographic locations. The overall objective of this study was to characterize the population structure of $S$. musiva across North America. The specific objectives were to (i) identify via whole-genome data genetic variants among geographically isolated populations across the North American range of the pathogen, (ii) estimate the degree of population structure within each of the sampled populations, (iii) characterize the connectivity among isolated populations of $S$. musiva, and (iv) identify the genomic regions with signals of local adaptation as a consequence of natural selection at the state or province level.

\section{RESULTS}

\section{S. musiva genome sequencing.}

To obtain genomic variants, $959,188,303$ reads were sequenced from genomic DNA of 122 isolates of $S$. musiva collected from symptomatic trees in plantations and natural stands across North America. Raw sequencing data were made available at NCBI BioProject PRJNA543887. Sequencing coverage varied across individual isolates from $17 \times$ to $80 \times$ (Supplementary Table S1). In total, 120,016 filtered singlenucleotide polymorphisms (SNPs) were obtained after filtering for variant quality across 13 chromosomes. Indels were omitted from the analysis.

\section{Population structure, individual phylogeny, and summary statistics.}

To identify the degree of population differentiation and evolutionary relationships across the geographic locations of $S$. musiva, we reconstructed a phylogenetic tree based on the 120,016 filtered biallelic SNPs. Low to moderate levels of genetic differentiation were detected across the sequenced strains of S. musiva. A maximum-likelihood (ML) phylogenetic reconstruction based on the identified SNPs revealed two main clades (Fig. 1). Clade 1 (southeastern United States [SE-US]) grouped isolates from West Virginia, Tennessee, Arkansas, Missouri, Kentucky, and three isolates from Pennsylvania. Clade 2 (US-CAN) grouped isolates from Alberta, British Columbia, Ontario, Quebec, Saskatchewan, North Dakota, Minnesota, Wisconsin, and one isolate from Pennsylvania (Fig. 1A). Within these two clades, a subset of the 18 British Columbia strains clustered with strong genetic differentiation assumed based on branch length (British Columbia 2 group [BC2]) (Fig. 1A). Low genetic differentiation across geographic populations was observed using Weir and Cockerham's $\mathrm{F}_{\mathrm{st}}$ (Weir and Cockerham 1984) (Supplementary Fig. S1), as well as significant differences on isolation by distance (IBD) (Mantel's test, $P<0.001)$. To predict the number of genetic clusters that represent the most plausible number of genetic groups for $S$. musiva in the United States and Canada, we evaluated the posterior probabilities for membership association with each geographic region per sample using a discriminant analysis of principal components (DAPC) (Jombart et al. 2010). The groupings from the DAPC support the clades identified in the ML phylogenetic reconstruction step described above (SE-US and US-CAN), with minimal evidence of withingroup differentiation (Fig. 1B). An exception was found for samples within the BC2 subset of British Columbia, which appeared to be its own distinct genetic group. No evidence of population structure by state was found in the phylogenetic reconstruction, DAPC clustering, or $\mathrm{F}_{\text {st }}$ estimation. This indicates that states or provinces do not represent genetic groups of S. musiva.

\section{Genetic clustering reveals a new subpopulation of $S$. musiva exclusive to British Columbia.}

To detect the number of distinct genetic clusters $(K)$ of $S$. musiva that may exist in North America, we reconstructed a DAPC without a priori population information and by estimating the optimal number of $\mathrm{K}$ based on the Bayesian information criterion (BIC). The optimal number of predictedgenetic clusters found by best $\mathrm{BIC}$ was $K=3\left(\mathrm{BIC}_{(K=3)}=\right.$ 1,012.921), named SE-US, US-CAN, and BC2 (Fig. 2). These three clusters represented the groups in which all populationscaled comparisons were performed. Clustering based on $K=4$ $\left(\mathrm{BIC}_{(K=4)}=1,013.155\right)$ and $K=5\left(\mathrm{BIC}_{(K=5)}=1,014.579\right)$ yielded a similar result, where three major groups were observed with different degrees of $K$ assignment for samples within the general US-CAN group (Supplementary Fig. S2).

Pairwise $\mathrm{F}_{\mathrm{st}}$ among the SE-US, US-CAN, and BC2 genetic clusters indicated low to moderate levels of genetic differentiation (Table 1) (Weir and Cockerham 1984). The $F_{\text {st }}$ values increased as the distance among predicted-genetic clusters 
increased. Migration rate between the three predicted-genetic clusters was also estimated using Migrate-n. There was evidence of gene flow among all predicted-genetic clusters (Fig. 3, inset). In Migrate-n, estimated $\theta$ indicated lower genetic diversity in SE-US $\left(\theta_{\text {mean }}=0.0058\right)$ and BC2 $\left(\theta_{\text {mean }}=0.0037\right)$ when compared with US-CAN $\left(\theta_{\text {mean }}=0.0176\right)$ (Fig. 3 , inset).

\section{Recombination in the North American population} of $S$. musiva.

To estimate the degree of recombination in S. musiva, we measured the estimated distribution of linkage via the standardized index of association $\left(\mathrm{I}_{\mathrm{A}}\right)$ (Brown et al. 1980) for each of the three predicted-genetic clusters (US-CAN, SE-US, and BC2)
A

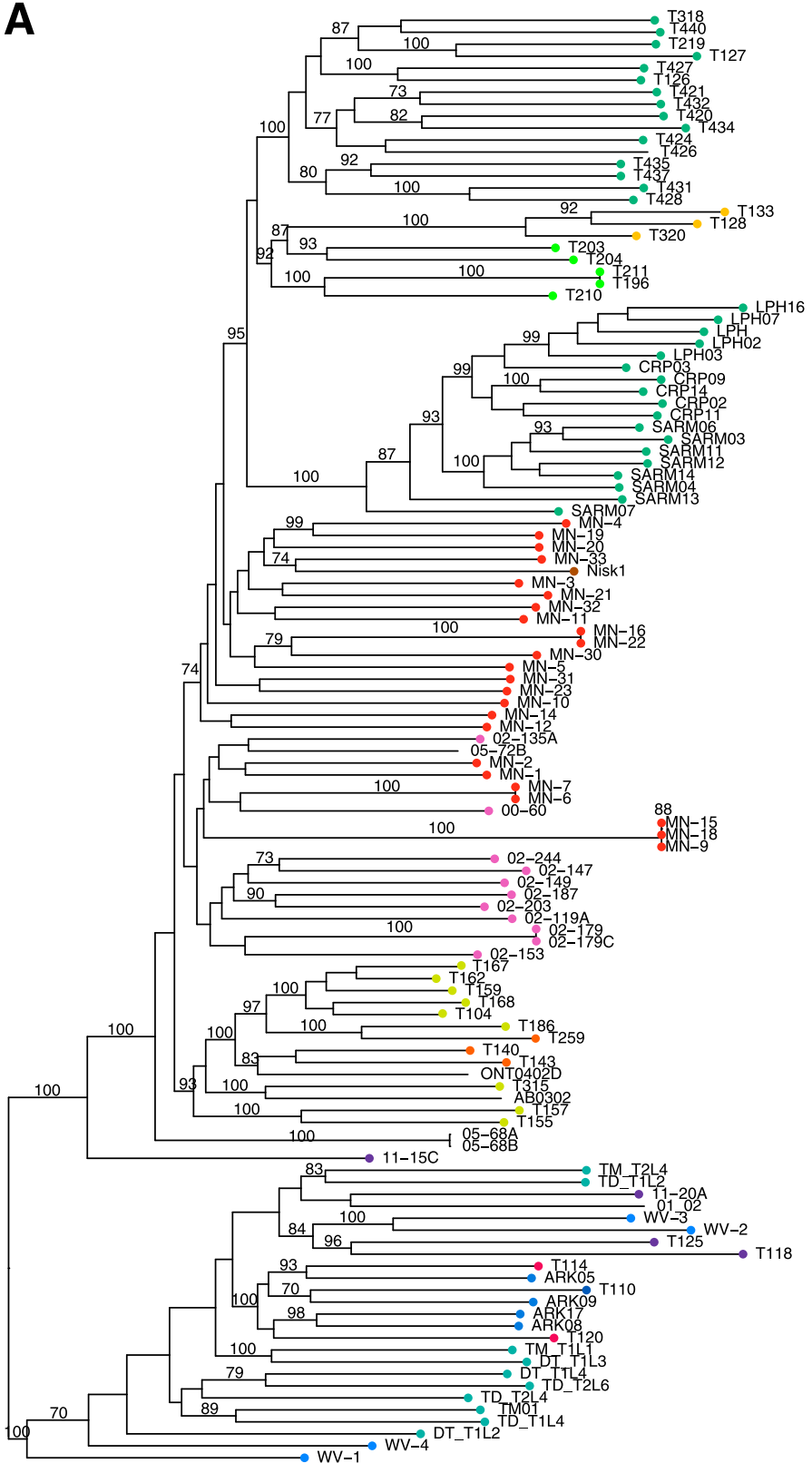

0.04 substitutions per site

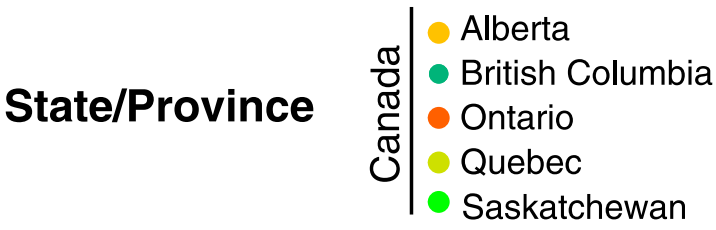

B

$\mathrm{BC} 2$

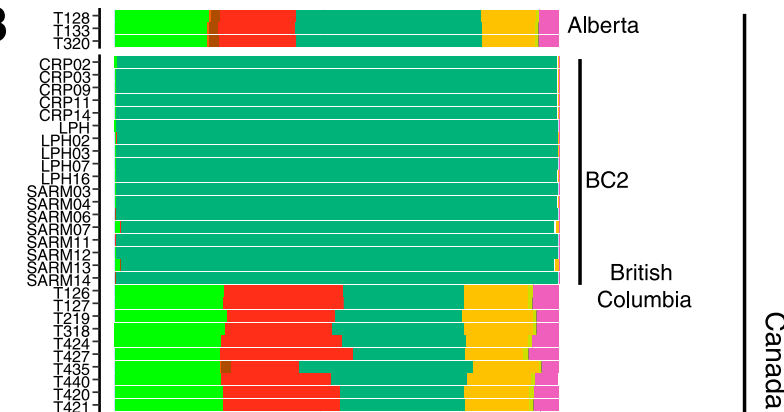

ڤ్య

Fig. 1. Population structure of Sphaerulina musiva across the United States and Canada. A, Maximum-likelihood tree reconstruction based on 120,016 singlenucleotide polymorphisms across the genomes of 122 isolates. Values above branches represent bootstrap support values after 1,000 bootstrap replicates. All values under 70 have been removed. Colored tips represent the state or province for each sample. B, Bar plot representing the posterior probability of membership to each state or province for all samples of S. musiva as calculated by a discriminant analysis of principal components in adegenet. Bar colors represent state or province. Each mark on the $y$ axis represents an isolate, while the colored bars represent the cumulative fraction of genetic material assigned to each sampled population. 
and compared them with simulations of populations with values of $\mathrm{I}_{\mathrm{A}}$ that ranged from 100 to $0 \%$ linkage. Extensive recombination was detected in the North American population of S. musiva. Significant differences across the various degrees of linkage and the different predicted-genetic clusters were observed (analysis of variance [ANOVA] df $=7, F=1708, P<$ 0.001). The distributions of the standardized $\mathrm{I}_{\mathrm{A}}$ for the SE-US and US-CAN genetic clusters were similar and fell within the 0 to $25 \%$ linkage simulation. This indicates a predominantly sexual mode of reproduction with a strong potential for recombination (Supplementary Fig. S3). In contrast, the distribution of the standardized $\mathrm{I}_{\mathrm{A}}$ for the $\mathrm{BC} 2$ genetic cluster was different from SE-US and US-CAN clusters, falling within the 25 to $50 \%$ range of the linkage simulations. The rate of linkage

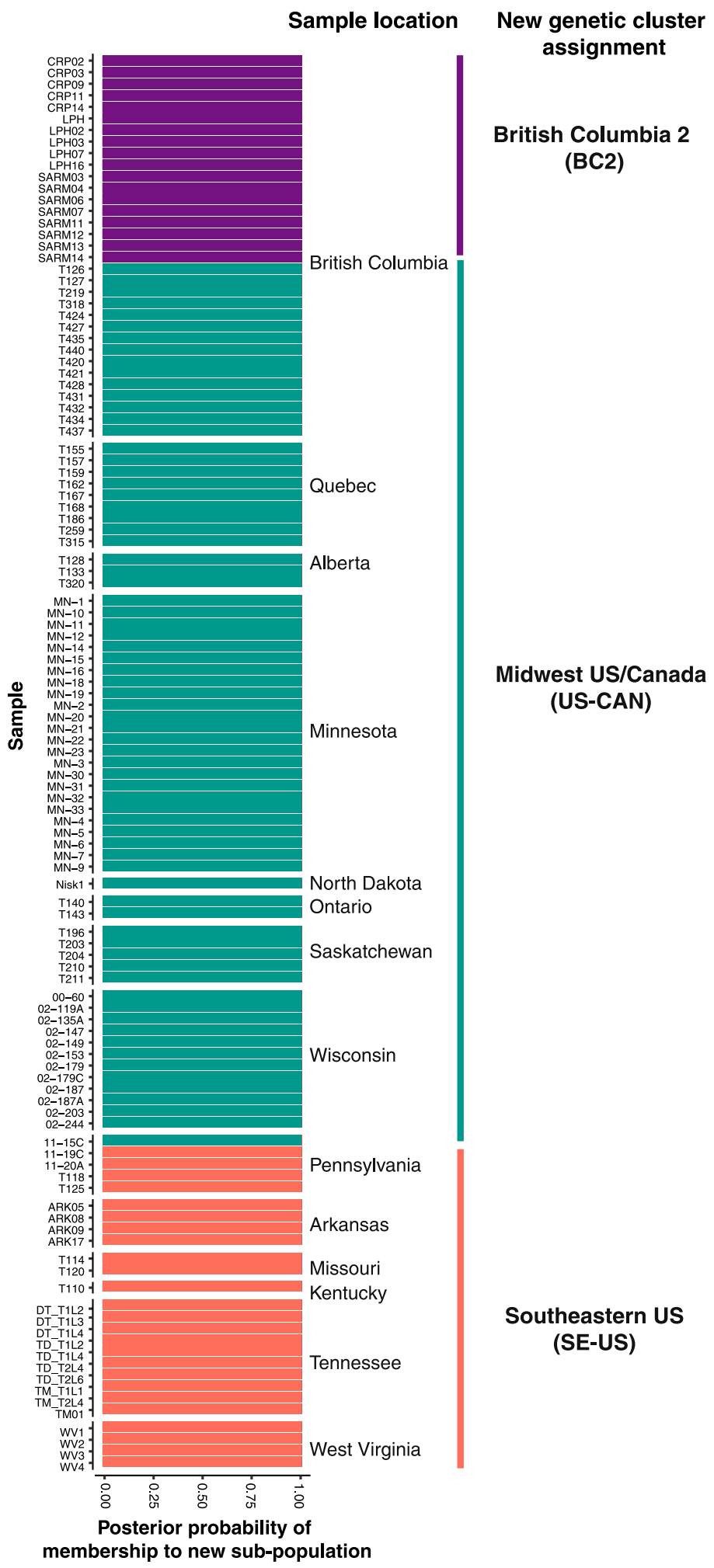

Fig. 2. Inference of population structure using discriminant analysis of principal components (adegenet) representing the most optimal number of clusters $(K=3)$ for 120,016 single-nucleotide polymorphisms across the genomes of 122 isolates of Sphaerulina musiva with population information. Each mark on the $y$ axis represents an isolate, while the colored bars represent the cumulative fraction of genetic material assigned to each new cluster. 
disequilibrium (LD) decay was estimated as the correlation coefficient $\left(r^{2}\right)$ between any two loci. The LD decay curves showed a similar rate of decay for the US-CAN and the SE-US genetic clusters, with a steep decline in the first hundred bases. Conversely, the LD decay showed that, at an $r^{2}$ of 0.2 , the distance is $24 \mathrm{bp}$ for US-CAN, $150 \mathrm{bp}$ for SE-US, and 4,000 bp for BC2, indicating a larger distance associated with LD in $\mathrm{BC} 2$ compared with the other genetic clusters (Supplementary Fig. S4).

\section{Local adaptation and selection}

in the North American population of $S$. musiva.

To test for evidence of local adaptation within the three predicted-genetic clusters of $S$. musiva, we screened variants for evidence of $F_{s t}$ outliers. No statistically significant $F_{s t}$ outliers were detected. We also searched for evidence of selection in genic regions by calculating the pairwise McDonald-Kreitman's test (MKT) (McDonald and Kreitman 1991) among all pairs of predicted-genetic clusters for all coding regions to detect genes that deviate from the expectation of neutral evolution. Six genes had a neutrality index $<1$, indicating plausible evidence for selection. However, Fisher's exact test did not support this hypothesis (Supplementary Table S2), where no evidence for selection was found.

\section{Genetic diversity of genes}

across $S$. musiva subpopulations.

We compared the genetic diversity across all predicted-genetic clusters to identify the degree of genomic differentiation within each genetic cluster. Genetic differentiation was measured by estimating the average number of pairwise nucleotide differences $(\pi)$ (Nei and Li 1979) and the haplotype diversity (Nei and Tajima 1981 ) for each gene model predicted in the genome of $S$. musiva. The overall estimation of genetic diversity via $\pi$ and haplotype diversity showed significant differences between the mean nucleotide diversity of BC2 and US-CAN/SE-US $\left(\pi_{\mathrm{BC} 2}=0.013\right.$, $\pi_{\mathrm{US}-\mathrm{CAN}}=0.021$, and $\left.\pi_{\mathrm{SE}-\mathrm{US}}=0.022 ; \chi^{2}=349.42, P<0.001\right)$ and the mean haplotype diversity of $\mathrm{BC} 2$ when compared with the other two genetic clusters $(\mathrm{BC} 2=0.264$, US-CAN $=0.434$, and SE-US $\left.=0.433 ; \chi^{2}=323.42, P<0.001\right)$. In addition, the number of monomorphic genes was also used to establish the degree of genetic differentiation across all predicted-genetic clusters. A higher number of monomorphic genes were discovered for BC2 compared with the other genetic clusters: $\mathrm{BC} 2=4,736(46 \%$ of the total genes in the $S$. musiva reference genome), US-CAN = $2,623(25 \%)$, and SE-US $=3,069(30 \%)\left(\chi^{2}=217.26, P<0.001\right)$. Pairwise nucleotide differences were estimated in effector coding-genes and carbohydrate-active enzymes (CAZy), two gene classes involved in plant-pathogen interactions. For the genetic diversity estimations across effector-coding genes and CAZy, 142 effector-coding genes were predicted using EffectorP (Supplementary Table S2) and $370 \mathrm{CAZy-coding} \mathrm{genes} \mathrm{were}$ recovered from the $S$. musiva reference genome. Differences between the mean of the distribution of $\pi$ for $\mathrm{BC} 2$ compared with the other two predicted-genetic clusters for effector-coding genes $\left(\pi_{\mathrm{BC} 2}=0.017, \pi_{\mathrm{US}-\mathrm{CAN}}=0.031\right.$, and $\pi_{\mathrm{SE}-\mathrm{US}}=0.016 ; \chi^{2}=20.086$, $P<0.001)$ and CAZy $\left(\pi_{\mathrm{BC} 2}=0.016, \pi_{\mathrm{US}-\mathrm{CAN}}=0.032\right.$, and $\pi_{\mathrm{SE}-\mathrm{US}}=$ 0.027; $\left.\chi^{2}=94.959, P<0.001\right)$ were detected.

\section{Phenotypic differences among genetic clusters of $S$. musiva in P. trichocarpa.}

To estimate differences in the degree of disease severity across all predicted-genetic clusters of S. musiva in North America, we measured the number of cankers per centimeter (CPC) and disease severity score (DSS) 3 weeks postinoculation of five genotypes of $P$. trichocarpa with all sequenced isolates of $S$. musiva. The relationships between
DSS/CPC and isolate, genetic cluster, and other variables were estimated using a linear mixed-effects model. The final model for CPC included the fixed effects of host genotype $(P<0.001)$ and genetic cluster $(P<0.001)$ and the random effects of block $(P<0.001)$ and isolate $(P<0.001)$ (Table 2$)$. The final model for DSS included the fixed effects of host genotype $(P<0.001)$, genetic cluster $(P<0.001)$ and the host genotype-genetic cluster interaction $(P<0.001)$, and the random effects of block $(P<0.001)$ and isolate $(P<0.001)$ (Table 2$)$. The BC2 predicted-genetic cluster had a lower mean disease severity for both DSS and CPC when compared with US-CAN and SE-US predicted-genetic clusters for all Populus genotypes (Fig. 4).

\section{DISCUSSION}

In contrast to the hypothesis of $S$. musiva following a migration-adaptation scenario for isolates collected at each

Table 1. Pairwise genetic dissimilarity between predicted-genetic clusters using Weir and Cockerman's $\mathrm{F}_{\text {st }}{ }^{\mathrm{a}}$

\begin{tabular}{lccc}
\hline Cluster & SE-US & US-CAN & BC2 \\
\hline SE-US & $\ldots$ & 0.12 & 0.19 \\
US-CAN & 0.12 & $\ldots$ & 0.10 \\
BC2 & 0.19 & 0.10 & $\ldots$ \\
\hline
\end{tabular}

${ }^{a}$ Isolates were from the southeastern United States (SE-US), the United States and Canada (US-CAN), and the new British Columbia cluster $(\mathrm{BC} 2)$.

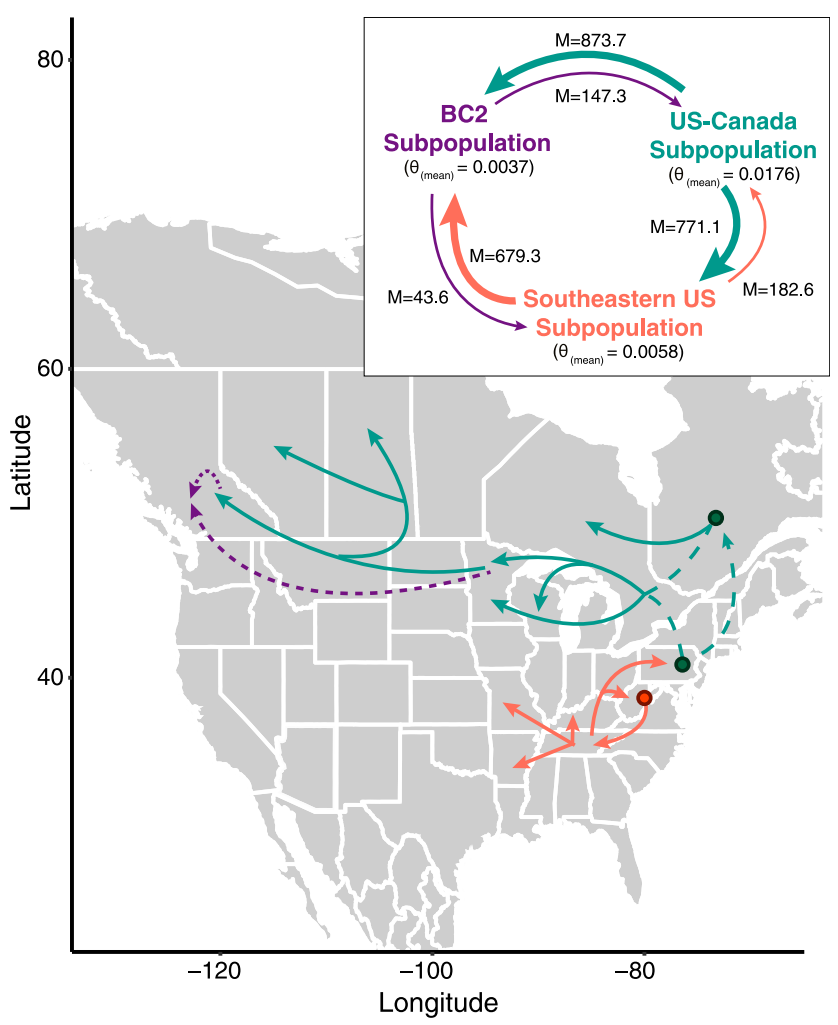

Fig. 3. Population dynamics of Sphaerulina musiva in the United States and Canada. The map represents the most plausible routes of migration of $S$. musiva between and across different populations. Colored circles surrounded by a black stroke represent the hypothesized center of origin for the United States-Canada (US-Canada) and the southeastern U.S. populations. $\mathrm{BC} 2=$ the new British Columbia cluster. Dashed lines represent equally plausible directions of dispersal of $S$. musiva. The inset represents the mean number of the mutation-scaled migrants (M) between subpopulations and the measure of $\theta$ per subpopulation. 
Populus plantation, our results indicate that $S$. musiva is genetically structured at a broader geographic scale. No genetic structure was found at a state or province level. However, consistently across all statistical tests, three different genetic clusters of $S$. musiva were identified grouping broad geographic regions: One of the clusters was made up of samples from SEUS, a second from samples collected in US-CAN, and a small cluster from British Columbia (BC2). These three genetic clusters likely represent three distinct evolutionary lineages of $S$. musiva in North America that were derived from multiple source populations. The movement of $S$. musiva into western North America likely occurred twice: once via West Virginia in the southeastern United States and once from Pennsylvania or Quebec (Fig. 3, black points). In addition, results show that Pennsylvania harbors isolates from distantly related clades and different membership probability, suggesting that it may be a center of contact between distinct evolutionary lineages of $S$. musiva. Both plausible source populations of $S$. musiva overlap with the native range of $P$. deltoides, the ancestral host of S. musiva (Feau et al. 2005; Sakalidis et al. 2016).

At a finer-scale IBD provides a potential explanation for the observed pattern of $S$. musiva in North America. Based on the assumed short range of dispersal of $S$. musiva spores, there was an expectation of significant IBD and high genetic differentiation across different geographic locations. The results do not fully support this expectation. Significant IBD is found in the Mantel's test; however, the degree of genetic differentiation varies between distantly separated states or provinces, with the largest $\mathrm{F}_{\mathrm{st}}$ values observed between northern and southern regions; for example, between Alberta and Kentucky $\left(\mathrm{F}_{\mathrm{st}}=0.33\right.$, $3,531 \mathrm{~km}$ apart $)$ or between Saskatchewan and Missouri $\left(\mathrm{F}_{\mathrm{st}}=\right.$ $0.21,2,483 \mathrm{~km}$ apart). In contrast, in an east-west direction, low genetic differentiation was estimated regardless of geographic distance; for example, between Pennsylvania and West Virginia $\left(F_{\mathrm{st}}=0.03,540 \mathrm{~km}\right.$ apart $)$ and between British Columbia and Quebec $\left(F_{\mathrm{st}}=0.08,3,972 \mathrm{~km}\right.$ apart $)$. The phylogenetic analysis supports this observation. The samples isolated from the U.S. Midwest (Minnesota, Wisconsin, and the single sample from North Dakota) are evolutionarily more similar to samples isolated from the eastern provinces of Canada (Ontario and Quebec). The phylogenetic reconstruction also shows that some samples from Alberta and Saskatchewan are closely related to a subset of isolates from British Columbia, forming an AlbertaSaskatchewan-British Columbia lineage. The cumulative evidence indicates plausible movement of the US-CAN genetic cluster in a northwesterly direction, as described by Sakalidis et al. (2016), who reported genetic similarities between $S$. musiva isolated from various geographic areas in western and central Canada.

Although significant IBD was found, as expected, the varying degree of genetic differentiation between geographic locations supports the hypothesis of anthropogenic-facilitated movement of S. musiva (Herath et al. 2016; Sakalidis et al. 2016). This hypothesis explains why low $F_{\text {st }}$ values are found between locations of Pennsylvania and West Virginia and between British Columbia and Quebec, whereas these locations are clustered in the same genetic cluster (Pennsylvania and West Virginia within SE-US and British Columbia and Quebec in US-CAN). This connectivity is seen not only across distant geographic locations but also across the different predictedgenetic clusters. High mutation-scaled migration rates across genetic clusters were observed, indicating that migration occurs predominantly from the most genetically diverse cluster (USCAN) (Fig. 3). This connectivity indicates high rates of gene flow via migration between genetic clusters and may be indicative of a high degree of rapid movement of this plant pathogen across large geographic regions. Human-mediated transport is the most plausible mechanism of dispersal in this case, similar to that of other plant pathogens (Chakraborty 2013; Goss et al. 2014; Kamvar et al. 2015b; Linde et al. 2009; Stukenbrock 2013; Stukenbrock et al. 2006; Tabima et al. 2018). Human-mediated movement of $S$. musiva has previously been described as the most likely mode of spread via the exchange of dormant cuttings among forest companies and breeding programs across North America (Abraham et al. 2018; Herath et al. 2016; Sakalidis et al. 2016). Latent infections in the vegetative cuttings may be the source of inoculum as new plantations are established (Abraham et al. 2018).

The discovery of a third smaller cluster in British Columbia (BC2), genetically distinct from the two large clusters (SE-US and US-CAN), is interesting. The monophyletic BC2 clade comprises highly similar isolates distinct from the AlbertaSaskatchewan-British Columbia lineage. BC2 has lower genetic diversity for all indices compared with the other clusters, a significantly higher number of monomorphic genes, lower recombination rates measured by $\mathrm{I}_{\mathrm{A}}$, a slower $\mathrm{LD}$ decay, and are less aggressive. In fact, $\mathrm{BC} 2$ may be a population of recent origin expanding from a single founder event. This hypothesis

Table 2. Linear mixed models using random effects for the phenotypic traits measured for Sphaerulina musiva isolates across North America ${ }^{\text {a }}$

\begin{tabular}{|c|c|c|c|c|c|c|c|}
\hline \multirow[b]{2}{*}{ Parameters, groups } & \multicolumn{5}{|c|}{ Fixed effects } & \multicolumn{2}{|c|}{ Random effects } \\
\hline & SS & Mean squares & DF & $\boldsymbol{F}$ & $\boldsymbol{P}$ & Variance & SD \\
\hline \multicolumn{8}{|l|}{$\overline{\mathrm{CPC}}$} \\
\hline \multicolumn{8}{|l|}{ Parameter } \\
\hline Population & 2.426 & 1.213 & 2 & 50.410 & $<0.001 * * *$ & $\ldots$ & $\ldots$ \\
\hline Genotype & 2.489 & 0.622 & 4 & 25.860 & $<0.001 * * *$ & $\ldots$ & $\ldots$ \\
\hline Population-genotype & 0.328 & 0.041 & 8 & 1.701 & $0.093 \mathrm{NS}$ & $\ldots$ & $\ldots$ \\
\hline \multicolumn{8}{|l|}{ Groups } \\
\hline Isolates & $\ldots$ & $\ldots$ & $\ldots$ & $\ldots$ & $\ldots$ & 0.005 & 0.069 \\
\hline Blocks & $\ldots$ & $\ldots$ & $\ldots$ & $\ldots$ & $\ldots$ & 0 & 0 \\
\hline Residual & $\ldots$ & $\ldots$ & $\ldots$ & $\ldots$ & $\ldots$ & 0.019 & 0.138 \\
\hline \multicolumn{8}{|l|}{ DSS } \\
\hline \multicolumn{8}{|l|}{ Parameter } \\
\hline Population & 25.725 & 12.862 & 2 & 9.994 & $<0.001 * * *$ & $\ldots$ & $\ldots$ \\
\hline Genotype & 212.073 & 53.018 & 4 & 41.196 & $<0.001 * * *$ & $\ldots$ & $\ldots$ \\
\hline Population-genotype & 51.579 & 6.447 & 8 & 5.010 & $<0.001 * * *$ & $\cdots$ & $\cdots$ \\
\hline \multicolumn{8}{|l|}{ Groups } \\
\hline Isolates & $\ldots$ & $\ldots$ & $\ldots$ & $\ldots$ & $\ldots$ & 0.544 & 0.738 \\
\hline Blocks & $\ldots$ & $\ldots$ & $\ldots$ & $\ldots$ & $\ldots$ & 0 & 0 \\
\hline Residual & $\ldots$ & $\ldots$ & $\ldots$ & $\ldots$ & $\ldots$ & 1.285 & 1.125 \\
\hline
\end{tabular}


is supported by the highly differentiated clade that comprises all of the BC2 samples: the single predicted-genetic cluster proposed by the DAPC approach, the prevalence of singlehaplotype genes in BC2 (17\%), and the relatively large number of genes when compared with the other genetic clusters that have $<1 \%$ of nonpolymorphic genes. The founder effect was likely followed by an expansion of this genetic cluster in the hybrid poplar plantations of south-central British Columbia, leading to a differentiated group of $S$. musiva different from other $S$. musiva populations that coinhabit British Columbia but share ancestry with isolates from Alberta and Saskatchewan. The likely source of this colonization is the US-CAN cluster. This assumption is based on the high mutation-scaled migration rate reflecting gene flow from US-CAN into BC2 (Fig. 3, inset) as well as the shared proportion of $\mathrm{BC} 2$ membership identified in samples of the Alberta-Saskatchewan-British Columbia lineage within the US-CAN genetic cluster (Fig. 1B). An alternative route of introduction of $\mathrm{BC} 2$ may be the SE-US. However, this result is not supported by the phylogenetic analysis, DAPC, or pairwise $\mathrm{F}_{\mathrm{st}}$ values. It is more likely that US-CAN served as a stepping stone from the SE-US cluster. The stepping-stone hypothesis explains the movement of other organisms, including the plant pathogen Alternaria alternata (Meng et al. 2018) and the brown algae Cystoseira amentacqe (Buonomo et al. 2017). This single colonization event of BC2 isolates into hybrid poplar plantations can also explain the low aggressiveness of this genetic cluster in the greenhouse assay of $P$. trichocarpa. Future endeavors will focus on understanding whether BC2 has a higher virulence in hybrid poplar or in other species of Populus, such as $P$. deltoides or P. balsamifera, other species of domesticated Populus commonly found in plantations.

The population genomic analysis of $S$. musiva identified broad regional genetic clusters, evidence for gene flow between these clusters, and interconnectedness of the North American $S$. musiva population across large geographic distances. The rates of immigration reflect greater connectivity best explained by human-mediated transport of infested plant material, reflecting the proposed mechanisms of dispersal reported by Sakalidis et al. (2016) and Abraham et al. (2018). Earlier studies indicated that IBD is only observed among a few geographically separate states or provinces and spore dispersal via wind is limited (Feau et al. 2005, 2010). The exchange of plant material among breeding programs has historically been a common practice in western Canada and the U.S. Midwest. Additionally, the discovery of a novel genetic cluster (BC2) with reduced aggressiveness on $P$. trichocarpa that is genetically distinct from other isolates in British Columbia from the US-CAN genetic cluster poses interesting questions about host specificity of $S$. musiva. Other mechanisms could be shaping these interactions such as the recent evolution of novel genes involved in pathogenicity, differences in gene expression, epigenetic relationships with aggressiveness, or host specificity. The tools used in this analysis do not allow for the exploration of these questions. Future analyses should be focused on identifying the genic or transcriptional elements that may be driving the phenotypic differences among genetic clusters of $S$. musiva in North America.

\section{MATERIALS AND METHODS}

\section{Population sampling, DNA extraction,} and genome sequencing.

In total, 122 isolates of $S$. musiva were isolated from Populus spp. and hybrids in plantations and natural stands across North America. Collections were made at various times over the last 20 years (Table 1; Supplementary Table S1). All isolations were conducted from cankers or from leaf spots. Initially, cankers were soaked for 2 min in a $5 \% \mathrm{NaClO}$ (bleach) solution and rinsed twice for $2 \mathrm{~min}$ with distilled water. The outer layer of the bark was scraped away from the stem and a 5-mm sliver of wood on the margin between necrotic and healthy tissue was removed. The sliver was placed on KV-8 medium (Stanosz and Stanosz 2002) with streptomycin and chloramphenicol. Pure cultures were stored in a $50 \%$ glycerol solution at $-80^{\circ} \mathrm{C}$.
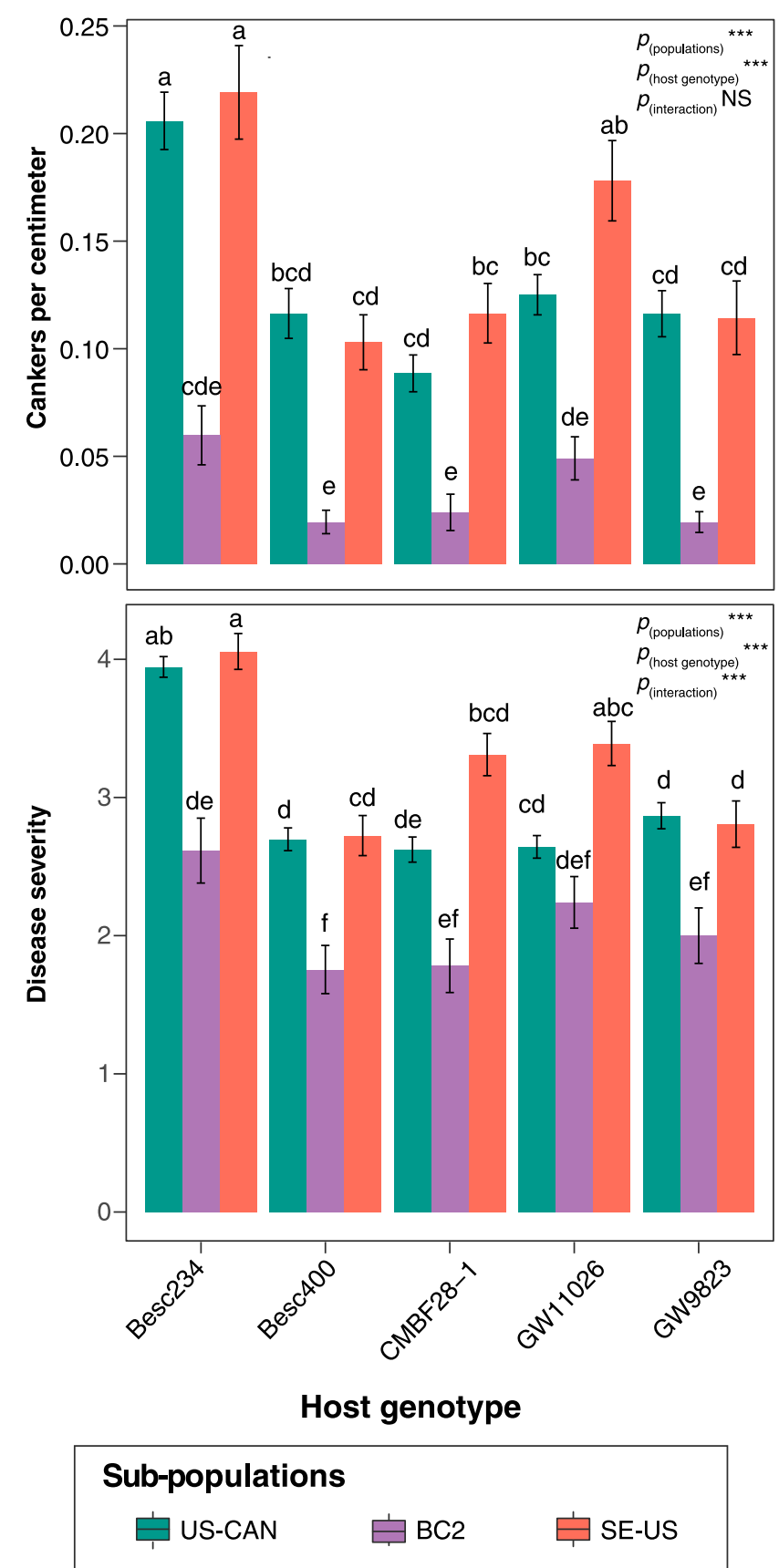

Fig. 4. Phenotypic differences between the three genetic clusters of Sphaerulina musiva following inoculation of five different genotypes of Populus trichocarpa. Boxplot diagrams representing A, number of cankers per genotype and $\mathbf{B}$, disease severity for each genetic cluster of $S$. musiva. Letters above each boxplot represent significant differences at $\alpha<0.01$ between each genetic cluster and genotype using Tukey honestly significant difference (A) or Dunn's test of multiple comparisons (B). Isolates were from the United States and Canada (US-CAN), the new British Columbia cluster (BC2), and the southeastern United States (SE-US). 
Isolates were taken out of cold storage, poured onto $\mathrm{KV}-8$ medium, and grown for 7 days under continuous light. Small pieces of sporulating mycelium were placed in sterile liquid $\mathrm{KV}-8$ media and grown for 5 days in the dark on a rotating shaker. Mycelium was collected by straining the liquid through Miracloth and measuring a 50-mg sample of tissue which was subsequently stored at $-80^{\circ} \mathrm{C}$. DNA extraction were conducted using the Thermo KingFisher Flex automated extraction system and the Omega Biotek's Mag-Bind Plant DNA DS 96 Kit at Oregon State University Center for Genome Research and Biocomputing (CGRB). Library preparation was conducted by the CGRB using the Wafergen DNA library prep. Size selection was done using BluePippin Targeted Size Selection for a 500bp insert size. The 122 genomes were sequenced using two lanes of Illumina HiSeq 3000 technology with 150-bp pairedend reads and a median insert size of $500 \mathrm{bp}$.

\section{Read mapping and quality filtering.}

Each FASTQ file per sequenced sample was aligned to the reference genome of $S$. musiva SO2202 v1.0 (Dhillon et al. 2015; Ohm et al. 2012) using bwa (Li and Durbin 2009). Variants were predicted using the GATK Haplotype Caller and saved as a variant caller format (VCF) file (McKenna et al. 2010). VCF files were filtered using the $v c f R$ 1.0.1 package (Knaus and Grünwald 2017) in R 3.5.1 (R Core Team 2017). Variants were filtered in two steps: (i) initial filtering removed variants with a read depth $<4 \times$ and a minimum threshold mapping quality of 44 and (ii) positions with $>40 \%$ missing data and a minimum allele frequency $<0.014$. This corresponds to alleles that are only present in two or fewer of the sequenced isolates. The filtered variants were used in the downstream analyses.

\section{Population structure of $S$. musiva across the United States and Canada.}

In order to determine whether genetic grouping corresponds with the geographic locations where isolates were collected, an ML tree was reconstructed using RAxML (1,000 bootstrap replicates; MULTIGAMMAI model of substitution) (Stamatakis 2014). In addition, the R packages poppr (Kamvar et al. 2014, 2015a) and adegenet (Jombart 2008) were used to perform a DAPC (Jombart et al. 2010), retaining 100 principal components and three discriminant components among the samples obtained from each of the geographic locations (state or province) in the study. Seven samples with no geographic information (01_02, AB0302, ONT0402D, T104, T167, T259, and T426) were removed from the following analyses. To determine the degree of genetic differentiation among geographic locations, we calculated a Mantel's test (Sokal 1979) using the mantel.rtest function at the ade4 R package (Dray and Dufour 2007), where the genetic distances calculated via bitwise distance were contrasted against the centroid of each state or province, and pairwise Weir and Cockerham's $\mathrm{F}_{\text {st }}$ (Weir and Cockerham 1984) between all geographic locations sampled using the assigner R package (Gosselin et al. 2019).

To predict the number of genetic clusters that represent the most plausible number of genetic groups for $S$. musiva in the United States and Canada, we used the find.clusters function in adegenet, retaining 100 principal components and three discriminant components. The find.clusters function used a maximum $K$ of 13, the same number of states or provinces from which samples in this study were isolated. To determine the best $K$ model, a selection of the best $K$ value was performed via BIC in the find.clusters function. A DAPC was performed across the new genetic clusters to determine the posterior probability of each isolate membership in the predicted cluster. These predicted-genetic clusters represented the new populations of S. musiva in North America and were used in all subsequent analyses.

To identify the degree of population differentiation between the newly predicted-genetic clusters for all pairwise combinations, differentiation was calculated via Weir and Cockerham's $\mathrm{F}_{\text {st }}$ (Weir and Cockerham 1984) in the $\mathrm{R}$ package assigner (Gosselin et al. 2019). Migrate-n was used to estimate the mutation scaled migration rate between genetic clusters, rates of mutation under mutation-drift equilibrium, and $\theta$ among the predicted-genetic clusters (Beerli and Felsenstein 2001). Migrate-N used the following parameters: 1,000,000 steps with 10 replicates, one hot chain, and three cold Monte Carlo Markov chain chains to estimate $\theta$ for each predicted-genetic cluster and migration rate between all plausible pairs of predicted-genetic clusters.

\section{Recombination in the North American population of $S$. musiva.}

Estimation of LD based on the distribution of the standardized $I_{A}$ (Brown et al. 1980) was performed for each of the three predicted-genetic clusters (US-CAN, SE-US, and BC2). The $\mathrm{I}_{\mathrm{A}}$ correlates with the predominant mode of reproduction as either sexual, clonal, or mixed. The function sample.ia from poppr (Kamvar et al. 2014) was used to calculate the standardized $\mathrm{I}_{\mathrm{A}}$ for 100,000 random variants. These values were used to estimate the distribution of standardized $\mathrm{I}_{\mathrm{A}}$ values. To determine the degree of $\mathrm{LD}$ for each cluster, we tested the difference between a simulated distribution of standardized $\mathrm{I}_{\mathrm{A}}$ and those estimated for the three genetic clusters. Simulations were conducted for five scenarios: $0,25,50,75$, and $100 \%$ linkage between 120,017 loci and 40 samples (median population size of the three clusters). These values represented degrees of sexual $(0 \%)$, clonal $(100 \%)$, and mixed $(25,50$, and $75 \%)$ modes of reproduction. Estimation of normality was performed using the Shapiro-Wilk's normality test for each of the distributions of standardized $\mathrm{I}_{\mathrm{A}}$ values for the observed and simulated data. An ANOVA was performed to test for significant differences among the distributions of the $\mathrm{I}_{\mathrm{A}}$ for the observed populations and the simulations. A Tukey's honestly significant difference (HSD) posthoc test was performed to test for differences among pairwise comparisons of the standardized $\mathrm{I}_{\mathrm{A}}$ among clusters and the simulated data sets. Tests were performed using the TukeyHSD function from R ( $\mathrm{R}$ Core Team 2017). The rate of LD decay was calculated for each population using the $r^{2}$ calculated in vcftools 0.1.15 (Danecek et al. 2011) with a maximum of $5 \mathrm{Mbp}$ between SNPs.

\section{Local adaptation, genetic diversity, and selection in the North American population of $S$. musiva.}

To search for genetic variants with evidence of selection within each predicted-genetic cluster, we identified $\mathrm{F}_{\mathrm{st}}$ outliers using BayesScan (Foll and Gaggiotti 2008) and a local calculation of Wright's $F_{\mathrm{st}}$ (Wright 1949). In addition, we extracted each gene model predicted for the $S$. musiva genome and estimated the neutrality index via the MKT (McDonald and Kreitman 1991) for each gene model between all pairs of predicted-genetic clusters. To calculate MKT, we extracted each annotated genic region (annotated as "gene" in the third column of the S. musiva S2202 v1.0 filtered model Genome Format File; available at the Joint Genome Institute). The DNA sequences were translated into protein using the revseq tool from EMBOSS (Rice et al. 2000) and each protein sequence was aligned for each pair of genetic clusters in MAFFT v7.401 (using the G-INS-I iterative refinement method) (Katoh and Standley 2013). We reverse-aligned each nucleotide sequence based on its protein alignment in order to maintain the integrity of the codon positions using RevTrans 1.4 (Wernersson and 
Pedersen 2003). MKT for all gene models was calculated using the PopGenome package in R (Pfeifer et al. 2014). Fisher's exact test was used to identify evidence for selection with genes with a neutrality index significantly different from 1.

In order to compare the genetic diversity among the three predicted-genetic clusters of $S$. musiva, the distribution of the average number of pairwise nucleotide differences $(\pi)$ (Nei and Li 1979) per gene were estimated for all the genes annotated in the $S$. musiva reference genome. Additionally, the pairwise nucleotide differences were estimated in two classes of genes involved in interactions between the pathogen and its host: effector coding-genes and CAZy. Putative effectors were predicted using the following criteria: (i) the gene was part of the secretome and (ii) the probability that the gene model codes for an effector protein. Secretome prediction was performed by estimating the probability of a signal peptide cleavage site for all predicted proteins in the $S$. musiva reference genome using SignalP 4.0 (Petersen et al. 2011). The criteria were a minimum threshold D-score $>0.8$. The $\mathrm{D}$-score probability reflects the ability to discriminate between a signal peptide signal versus a nonsignal peptide signal. The sequences of the reduced list of putative secreted proteins was analyzed using EffectorP 2.0 to predict genes which encoded effectors (Sperschneider et al. 2016). EffectorP 2.0 uses machine learning to predict the probability that a secreted protein is an effector. All proteins with a probability $>0.5$ were considered putative effectors (Supplementary Table S2). CAZy-encoding genes were identified using the annotations from the $S$. musiva reference genome (Dhillon et al. 2015; Ohm et al. 2012). The predicted effector and annotated CAZy-encoding genes were used in the subsequent analysis. Estimates of $\pi$ were made using the nuc.div function of the pegas $\mathrm{R}$ package (Paradis 2010). The estimates of $\pi$ were compared among predicted-genetic clusters for effectors and CAZy-encoding genes using a Kruskal-Wallis rank test of the distribution of estimated values of $\pi$.

Haplotype diversity (Nei and Tajima 1981) was estimated as an independent measurement of genetic diversity for all of the predicted gene models of $S$. musiva using the hap.div function from the pegas $\mathrm{R}$ package (Paradis 2010).

\section{Phenotypic differences among genetic clusters.}

Dormant $P$. trichocarpa cuttings from five different genotypes (BESC-234, BESC-400, CMBF-28-1, GW-11026, and GW-9823) were collected from a common garden at Oregon State University (Evans et al. 2014). The cuttings were prepared, grown, and fertilized as described by Dunnell and LeBoldus (2017). Inoculations took place after trees reached a minimum height of $30 \mathrm{~cm}$, approximately 4 months after the cuttings were planted. The trees were inoculated by spraying them with a spore suspensions of $1 \times 10^{6}$ spores $/ \mathrm{ml}$ (LeBoldus et al. 2010). Each spore suspension was sprayed on four replicates of each of five genotypes of $P$. trichocarpa (total trees per isolate $=20$; tree height approximately $30 \mathrm{~cm}$ ). Inoculated trees were placed in black plastic bags and incubated at room temperature for $48 \mathrm{~h}$. The trees were then removed from the bags and grown with an 18-h photoperiod supplemented with artificial lights. After 3 weeks and prior to sporulation, the DSS (on a scale of 1 to 5) (Dunnell and LeBoldus 2017) and CPC were determined. Tree height was measured at the time of inoculation.

A linear mixed-effects model using the nlme package (Pinheiro et al. 2019) in $\mathrm{R}$ was used to analyze the effect of treatment on DSS and CPC. Statistical significance was assessed at $\alpha=0.05$. Model selection was conducted using a likelihood ratio $\chi^{2}$ test to determine the significance of each of the parameters including block, predicted-genetic cluster, isolate, and host genotype-predicted-genetic cluster. The order of testing was based on the default $z$-test performed in R. In each subsequent model, nonsignificant parameters were removed. The same analysis was conducted for the two parameters DSS and CPC separately. Tukey's HSD was calculated in R for each phenotypic trait to determine which predicted-genetic cluster had a statistically lower aggressiveness.

The initial model for both parameters was $Y_{i j k l}=\mu+G_{j}+C_{k}+$ $\left(G_{j} \times C_{k}\right)+\beta_{i}+I_{j}+\varepsilon_{i j k}$, where $Y_{i j k}$ is the DSS or CPC value in the $i$ th block for the $l$ th host genotype nested within the $k$ th genetic cluster inoculated with the $j$ th isolate, $\mu$ is the overall mean, $G_{j}$ is the fixed effect of the host genotype, $C_{k}$ is the fixed effect of the genetic cluster, $G_{j} \times C_{k}$ is the interaction between host genotype and genetic cluster in the response variable, $\beta_{i}$ is the random effect of the $i$ th block, $i=1$ to $4, I_{j}$ is the random effect of the $j$ th isolate, $j=1$ to 212 , and $\varepsilon_{i j k}$ is the residual error.

\section{ACKNOWLEDGMENTS}

We thank G. Stanosz for the contribution of isolates used in this study.

\section{LITERATURE CITED}

Abraham, N. D., Chitrampal, P., Keriö, S., and LeBoldus, J. M. 2018. Multiplex qPCR for detection and quantification of Sphaerulina musiva in Populus stems. Plant Pathol. 67:1874-1882.

Beerli, P., and Felsenstein, J. 2001. Maximum likelihood estimation of a migration matrix and effective population sizes in $n$ subpopulations by using a coalescent approach. Proc. Natl. Acad. Sci. U.S.A. 98: 4563-4568

Bier, J. E. 1939. Septoria canker of introduced and native hybrid poplars. Can. J. Res. 17c:195-204.

Brown, A. H. D., Feldman, M. W., and Nevo, E. 1980. Multilocus structure of natural populations of Hordeum spontaneum. Genetics 96:523-536.

Buonomo, R., Assis, J., Fernandes, F., Engelen, A. H., Airoldi, L., and Serrão, E. A. 2017. Habitat continuity and stepping-stone oceanographic distances explain population genetic connectivity of the brown alga Cystoseira amentacea. Mol. Ecol. 26:766-780.

Callan, B., Leal, I., Foord, B., Dennis, J., and Van Oosten, C. 2007. Septoria musiva isolated from cankered stems in hybrid poplar stool beds, Fraser Valley, British Columbia. N. Am. Fungi 2:1-9.

Chakraborty, S. 2013. Migrate or evolve: Options for plant pathogens under climate change. Glob. Change Biol. 19:1985-2000.

Couch, B. C., Fudal, I., Lebrun, M.-H., Tharreau, D., Valent, B., van Kim, P., Nottéghem, J.-L., and Kohn, L. M. 2005. Origins of host-specific populations of the blast pathogen Magnaporthe oryzae in crop domestication with subsequent expansion of pandemic clones on rice and weeds of rice. Genetics 170:613-630.

Danecek, P., Auton, A., Abecasis, G., Albers, C. A., Banks, E., DePristo, M. A., Handsaker, R. E., Lunter, G., Marth, G. T., Sherry, S. T., McVean, G., Durbin, R., and 1000 Genomes Project Analysis Group. 2011. The variant call format and VCFtools. Bioinformatics 27:2156-2158.

Dhillon, B., Feau, N., Aerts, A. L., Beauseigle, S., Bernier, L., Copeland, A., Foster, A., Gill, N., Henrissat, B., Herath, P., LaButti, K. M., Levasseur, A., Lindquist, E. A., Majoor, E., Ohm, R. A., Pangilinan, J. L., Pribowo, A., Saddler, J. N., Sakalidis, M. L., de Vries, R. P., Grigoriev, I. V., Goodwin, S. B., Tanguay, P., and Hamelin, R. C. 2015. Horizontal gene transfer and gene dosage drives adaptation to wood colonization in a tree pathogen. Proc. Natl. Acad. Sci. U.S.A. 112:3451-3456.

Dray, S. and Dufour, A.-B. 2007. The ade4 Package: Implementing the duality diagram for ecologists. J. Stat. Softw. 22:1-20.

Dunnell, K. L., and LeBoldus, J. M. 2017. The correlation between Septoria leaf spot and stem canker resistance in hybrid poplar. Plant Dis. 101: 464-469.

Evans, L. M., Slavov, G. T., Rodgers-Melnick, E., Martin, J., Ranjan, P., Muchero, W., Brunner, A. M., Schackwitz, W., Gunter, L., Chen, J.-G., Tuskan, G. A., and DiFazio, S. P. 2014. Population genomics of Populus trichocarpa identifies signatures of selection and adaptive trait associations. Nat. Genet. 46:1089-1096.

Feau, N., Hamelin, R. C., Vandecasteele, C., Stanosz, G. R., and Bernier, L. 2005. Genetic Structure of Mycosphaerella populorum (Anamorph Septoria musiva) Populations in North-Central and Northeastern North America. Phytopathology 95:608-616.

Feau, N., Mottet, M.-J., Périnet, P., Hamelin, R. C., and Bernier, L. 2010. Recent advances related to poplar leaf spot and canker caused by Septoria musiva. Can. J. Plant Pathol. 32:122-134. 
Foll, M., and Gaggiotti, O. 2008. A genome-scan method to identify selected loci appropriate for both dominant and codominant markers: A Bayesian perspective. Genetics 180:977-993.

García-Palacios, P., Milla, R., Delgado-Baquerizo, M., Martín-Robles, N., Alvaro-Sánchez, M., and Wall, D. H. 2013. Side-effects of plant domestication: Ecosystem impacts of changes in litter quality. New Phytol. 198:504-513.

Giraud, T., Gladieux, P., and Gavrilets, S. 2010. Linking the emergence of fungal plant diseases with ecological speciation. Trends Ecol. Evol. 25: 387-395.

Goss, E. M., Tabima, J. F., Cooke, D. E., Restrepo, S., Fry, W. E., Forbes, G. A., Fieland, V. J., Cardenas, M., and Grünwald, N. J. 2014. The Irish potato famine pathogen Phytophthora infestans originated in central Mexico rather than the Andes. Proc. Natl. Acad. Sci. U.S.A. 111:8791-8796.

Gosselin, T., Anderson, C. F., Ferchaud, A., and Bar, I. 2019. thierrygosselin/ assigner: Assigner 0.5.6 2019-05-01. https://zenodo.org/record/2656728

Gyenis, L., Anderson, N. A., and Ostry, M. E. 2003. Biological control of Septoria leaf spot disease of hybrid poplar in the field. Plant Dis. 87: 809-813.

Herath, P., Beauseigle, S., Dhillon, B., Ojeda, D. I., Bilodeau, G., Isabel, N., Gros-Louis, M.-C., Kope, H., Zeglen, S., Hamelin, R. C., and Feau, N. 2016. Anthropogenic signature in the incidence and distribution of an emerging pathogen of poplars. Biol. Invas. 18:1147-1161.

Jombart, T. 2008. adegenet: A R package for the multivariate analysis of genetic markers. Bioinformatics 24:1403-1405.

Jombart, T., Devillard, S., and Balloux, F. 2010. Discriminant analysis of principal components: A new method for the analysis of genetically structured populations. BMC Genet. 11:94.

Kamvar, Z. N., Brooks, J. C., and Grünwald, N. J. 2015a. Novel R tools for analysis of genome-wide population genetic data with emphasis on clonality. Front. Genet. 6:208.

Kamvar, Z. N., Larsen, M. M., Kanaskie, A. M., Hansen, E. M., and Grünwald, N. J. 2015b. Spatial and temporal analysis of populations of the sudden oak death pathogen in Oregon forests. Phytopathology 105 982-989.

Kamvar, Z. N., Tabima, J. F., and Grünwald, N. J. 2014. Poppr: An R package for genetic analysis of populations with clonal, partially clonal, and/or sexual reproduction. PeerJ 2:e281.

Katoh, K., and Standley, D. M. 2013. MAFFT multiple sequence alignment software version 7: Improvements in performance and usability. Mol. Biol. Evol. 30:772-780.

Knaus, B. J., and Grünwald, N. J. 2017. vcfr: A package to manipulate and visualize variant call format data in R. Mol. Ecol. Resour. 17:44-53.

LeBoldus, J. M., Blenis, P. V., and Thomas, B. R. 2010. A method to induce stem cankers by inoculating nonwounded Populus clones with Septoria musiva spore suspensions. Plant Dis. 94:1238-1242.

LeBoldus, J. M., Blenis, P. V., Thomas, B. R., Feau, N., and Bernier, L. 2009. Susceptibility of Populus balsamifera to Septoria musiva: A field study and greenhouse experiment. Plant Dis. 93:1146-1150.

Li, H., and Durbin, R. 2009. Fast and accurate short read alignment with Burrows-Wheeler transform. Bioinformatics 25:1754-1760.

Linde, C. C., Zala, M., and McDonald, B. A. 2009. Molecular evidence for recent founder populations and human-mediated migration in the barley scald pathogen Rhynchosporium secalis. Mol. Phylogenet. Evol. 51: 454-464.

McDonald, J. H., and Kreitman, M. 1991. Adaptive protein evolution at the Adh locus in Drosophila. Nature 351:652-654.

McKenna, A., Hanna, M., Banks, E., Sivachenko, A., Cibulskis, K., Kernytsky, A., Garimella, K., Altshuler, D., Gabriel, S., Daly, M., and DePristo, M. A. 2010. The Genome Analysis Toolkit: A MapReduce framework for analyzing next-generation DNA sequencing data. Genome Res. 20:1297-1303.

Meng, J.-W., He, D.-C., Zhu, W., Yang, L.-N., Wu, E.-J., Xie, J.-H., Shang, L.-P., and Zhan, J. 2018. Human-mediated gene flow contributes to metapopulation genetic structure of the pathogenic fungus Alternaria alternata from potato. Front. Plant Sci. 9:198.

Muchero, W., Sondreli, K. L., Chen, J.-G., Urbanowicz, B. R., Zhang, J., Singan, V., Yang, Y., Brueggeman, R. S., Franco-Coronado, J., Abraham, N., Yang, J.-Y., Moremen, K. W., Weisberg, A. J., Chang, J. H., Lindquist, E., Barry, K., Ranjan, P., Jawdy, S., Schmutz, J., Tuskan, G. A., and LeBoldus, J. M. 2018. Association mapping, transcriptomics, and transient expression identify candidate genes mediating plant-pathogen interactions in a tree. Proc. Natl. Acad. Sci. U.S.A. 115:11573-11578.

Nei, M., and Li, W. H. 1979. Mathematical model for studying genetic variation in terms of restriction endonucleases. Proc. Natl. Acad. Sci. U.S.A. 76:5269-5273.
Nei, M., and Tajima, F. 1981. Genetic drift and estimation of effective population size. Genetics 98:625-640.

Ohm, R. A., Feau, N., Henrissat, B., Schoch, C. L., Horwitz, B. A., Barry, K. W., Condon, B. J., Copeland, A. C., Dhillon, B., Glaser, F., Hesse, C. N., Kosti, I., LaButti, K., Lindquist, E. A., Lucas, S., Salamov, A. A., Bradshaw, R. E., Ciuffetti, L., Hamelin, R. C., Kema, G. H. J., Lawrence, C., Scott, J. A., Spatafora, J. W., Turgeon, B. G., de Wit, P. J. G. M., Zhong, S., Goodwin, S. B., and Grigoriev, I. V. 2012. Diverse lifestyles and strategies of plant pathogenesis encoded in the genomes of eighteen Dothideomycetes fungi. PLoS Pathog. 8:e1003037.

Ostry, M. E., and McNabb, H. S. 1985. Susceptibility of Populus species and hybrids to disease in the north central United States. Plant Dis. 69: 755-757.

Ostry, M. E., and McNabb, H. S. 1990. Minimizing disease injury to hybrid poplars. J. Environ. Hortic. 8:96-98.

Ostry, M. E., McNabb, H. S., Jr., and Moore, L. M. 1989. A Guide to Insect, Disease, and Animal Pests of Poplars. Agriculture Handbook 677. United States Department of Agriculture Forest Service.

Paradis, E. 2010. pegas: An R package for population genetics with an integrated-modular approach. Bioinformatics 26:419-420.

Peck, C. H. 1881. New species of fungi. Bot. Gaz. 6:274-279.

Petersen, T. N., Brunak, S., von Heijne, G., and Nielsen, H. 2011. SignalP 4.0: Discriminating signal peptides from transmembrane regions. Nat. Methods 8:785-786.

Pfeifer, B., Wittelsbürger, U., Ramos-Onsins, S. E., and Lercher, M. J. 2014. PopGenome: An efficient Swiss army knife for population genomic analyses in R. Mol. Biol. Evol. 31:1929-1936.

Pinheiro, J., Bates, D., DebRoy, S., Sarkar, D., and R Core Team. 2019. nlme: Linear and Nonlinear Mixed Effects Models. R package version 3.1-141. https://cran.r-project.org/web/packages/nlme/index.html

Qin, R., and LeBoldus, J. M. 2014. The infection biology of Sphaerulina musiva: Clues to understanding a forest pathogen. PLoS One 9:e103477.

R Core Team. 2017. R: A Language and Environment for Statistical Computing. R Foundation for Statistical Computing, Vienna, Austria.

Rice, P., Longden, I., and Bleasby, A. 2000. EMBOSS: The European Molecular Biology Open Software Suite. Trends Genet. 16:276-277.

Sakalidis, M. L., Feau, N., Dhillon, B., and Hamelin, R. C. 2016. Genetic patterns reveal historical and contemporary dispersal of a tree pathogen. Biol. Invas. 18:1781-1799.

Santos, Á. F. dos, Machado, E. B., Stanosz, G. R., and Smith, D. R. 2010. Primeiro relato da ocorrência de Septoria musiva em álamo no Brasil [First report of Septoria musiva in poplar in Brazil]. Trop. Plant Pathol. 35:052-053. doi:

Sarasola, A. A. 1944. Dos septoriosis de las alamedas Argentinas. Rev. Argent. Agron. 11:20-43.

Sokal, R. R. 1979. Testing statistical significance of geographic variation patterns. Syst. Zool. 28:227-232.

Sperschneider, J., Gardiner, D. M., Dodds, P. N., Tini, F., Covarelli, L., Singh, K. B., Manners, J. M., and Taylor, J. M. 2016. EffectorP Predicting fungal effector proteins from secretomes using machine learning. New Phytol. 210:743-761.

Stamatakis, A. 2014. RAxML version 8: A tool for phylogenetic analysis and post-analysis of large phylogenies. Bioinformatics 30:1312-1313.

Stanosz, J. C., and Stanosz, G. R. 2002. A medium to enhance identification of Septoria musiva from poplar cankers. For. Pathol. 32:145-152.

Stanturf, J. A., and Zhang, D. 2003. Plantations forests in the United States of America: Past, present and future. http://www.fao.org/3/xii/0325b1.htm

Stukenbrock, E. H. 2013. Evolution, selection and isolation: A genomic view of speciation in fungal plant pathogens. New Phytol. 199:895-907.

Stukenbrock, E. H., Banke, S., and McDonald, B. A. 2006. Global migration patterns in the fungal wheat pathogen Phaeosphaeria nodorum. Mol. Ecol. 15:2895-2904.

Tabima, J. F., Coffey, M. D., Zazada, I. A., and Grünwald, N. J. 2018. Populations of Phytophthora rubi show little differentiation and high rates of migration among states in the western United States. Mol. PlantMicrobe Interact. 31:614-622.

Tuskan, G. A., Difazio, S., Jansson, S., Bohlmann, J., Grigoriev, I., Hellsten, U., Putnam, N., Ralph, S., Rombauts, S., Salamov, A., Schein, J., Sterck, L., Aerts, A., Bhalerao, R. R., Bhalerao, R. P., Blaudez, D., Boerjan, W. Brun, A., Brunner, A., Busov, V., Campbell, M., Carlson, J., Chalot, M., Chapman, J., Chen, G.-L., Cooper, D., Coutinho, P. M., Couturier, J., Covert, S., Cronk, Q., Cunningham, R., Davis, J., Degroeve, S., Déjardin, A., Depamphilis, C., Detter, J., Dirks, B., Dubchak, I., Duplessis, S. Ehlting, J., Ellis, B., Gendler, K., Goodstein, D., Gribskov, M., Grimwood, J., Groover, A., Gunter, L., Hamberger, B., Heinze, B., Helariutta, Y. Henrissat, B., Holligan, D., Holt, R., Huang, W., Islam-Faridi, N., Jones, S., Jones-Rhoades, M., Jorgensen, R., Joshi, C., Kangasjärvi, J., Karlsson, 
J., Kelleher, C., Kirkpatrick, R., Kirst, M., Kohler, A., Kalluri, U., Larimer, F., Leebens-Mack, J., Leplé, J.-C., Locascio, P., Lou, Y., Lucas, S., Martin, F., Montanini, B., Napoli, C., Nelson, D. R., Nelson, C., Nieminen, K., Nilsson, O., Pereda, V., Peter, G., Philippe, R., Pilate, G., Poliakov, A., Razumovskaya, J., Richardson, P., Rinaldi, C., Ritland, K., Rouzé, P., Ryaboy, D., Schmutz, J., Schrader, J., Segerman, B., Shin, H., Siddiqui, A., Sterky, F., Terry, A., Tsai, C.-J., Uberbacher, E., Unneberg, P., Vahala, J., Wall, K., Wessler, S., Yang, G., Yin, T., Douglas, C., Marra, M., Sandberg, G., Van de Peer, Y., and Rokhsar, D. 2006. The genome of black cottonwood, Populus trichocarpa (Torr. \& Gray). Science 313:1596-1604.

Waterman, A. M. 1946. Canker of hybrid poplar clones in the United States, caused by Septoria musiva. Phytopathology 36:148-156.
Waterman, A. M. 1955. Septoria Canker of Poplars in the United States. United States Department of Agriculture, Washington, DC, U.S.A.

Weir, B. S., and Cockerham, C. C. 1984. Estimating F-statistics for the analysis of population structure. Evolution 38:1358-1370.

Wernersson, R., and Pedersen, A. G. 2003. RevTrans: Multiple alignment of coding DNA from aligned amino acid sequences. Nucleic Acids Res. 31 3537-3539.

Wright, S. 1949. The genetical structure of populations. Ann. Eugen. 15: 323-354.

Zeder, M. A. 2008. Domestication and early agriculture in the Mediterranean Basin: Origins, diffusion, and impact. Proc. Natl. Acad. Sci. U.S.A. 105:11597-11604. 